\title{
Evaluating the effects of statistical changes on internal dosimetry
}

\author{
Z. Sajjadi ${ }^{1 \star}$, H. Miri-Hakimabad ${ }^{2}$, L. Rafat-Motavalli ${ }^{2}$ and S. Mohammadi ${ }^{1}$ \\ 1 Department of Physics, Payame Noor University (PNU), 19395-3697, Tehran, Iran. \\ 2 Department of Physics, School of Sciences, Ferdowsi University of Mashhad, 91775-1436, Mashhad, Iran.
}

Received 2 October 2014 - Accepted 26 May 2015

\begin{abstract}
Currently, internal dosimetry evaluations are performed using reference computational phantoms. There are significant discrepancies in the organ absorbed doses regarding the variations in organ mass. In this study, to investigate the effects of changes in the lung mass on the results of internal dosimetry, 98 similar mathematical phantoms were developed, so that the masses of their lungs changed with a Gaussian distribution. The lung was selected as the source. Doses delivered to the organs/tissues for photons with different energy levels, and also per decay of ${ }^{131} \mathrm{I}$, were calculated using MCNPX. The results showed that changing the mass of the lung has effects on the dose of the lung, especially for low-energy photons and electrons resulting from the decay of ${ }^{131} \mathrm{I}$. According to the statistical distribution in terms of the SAF as a function of the lung mass, the average value of organ SAFs and the coefficient of variations were estimated. The uncertainties of the lung SAF due to the lung mass variation can be described by the coefficient of variation (CV), which changed from $9 \%$ to $19 \%$. This occurs for photon energy in the range of 10 to $4000 \mathrm{keV}$. This figure stands at $18 \%$ per decay of ${ }^{131} \mathrm{I}$.
\end{abstract}

Keywords: statistical phantom / internal dosimetry / Monte Carlo / organ dose

\section{Introduction}

In nuclear medicine, small amounts of radioactively labeled drugs (radiopharmaceuticals) are injected into the bloodstream and are then directly incorporated within the tissues being imaged or treated. Absorbed dose calculations are needed to evaluate the risks involved in using radiopharmaceuticals for both imaging and, more importantly, radionuclide therapy (Han, 2005). It is not possible to measure doses directly in the living body. By developing computational phantoms (anatomical models of the human body) and using Monte Carlo calculations, one can estimate the amount of the received dose in different diagnostic and treatment procedures. In dosimetric calculations of internal radiation therapy, reference phantoms are used, which are developed based on the anatomical data of 50th percentiles of a population (ICRP, 1975, 2002; $\mathrm{Xu}$ and Eckerman, 2009). According to the tomographic images, the shapes and sizes of internal organs of the human body differ significantly from one person to another (Mofrad et al., 2010; Ebrahimi-Khankook et al., 2015). There are many reference phantoms developed by different groups and some sets of patient-dependent phantoms. These different reference phantoms can be significantly different in the amount of the delivered dose, because their initial anatomies are based on different people (Xu and Liu, 2011). It is worth noting that estimating the exact amount of the dose helps to optimize the therapy in

\footnotetext{
^h_sajadi@pnu.ac.ir
}

nuclear medicine. It is in this quest that performing patientindividualized dose calculations is of vital importance (Stabin, 2013).

One way of estimating the exact amount of the dose is developing patient-specific phantoms by imaging their entire bodies. This is a very time-consuming approach $(\mathrm{Xu}$ and Eckerman, 2009) and thus not achievable now, because the patient's treatment should not be postponed. Instead, it is a good idea to use software such as MIRDOSE3 and OLINDA/EXM to calculate the absorbed dose in which phantoms of various ages and the common radiopharmaceuticals are defined (Williams et al., 2002; Stabin et al., 2005). Although it is possible to do mass correction for the dose calculation, in most clinical centers, a similar amount of dose is administered for different patients (Chen et al., 2005; Yu et al., 2006). In this regard, we calculate the uncertainty regarding mass variations and clarify the contributions of electrons and photons to this uncertainty. We investigate to what extent mass correction reduces the uncertainty.

In the third international workshop of computational phantoms for radiation protection diagnostic and radiotherapy procedures, held in August 2011 in China, Professor Xu introduced a new method for external dosimetry calculations. He suggested that a library containing all the amounts of absorbed doses of available phantoms should be created. In this way, changing the characteristics of the anatomical organs based on a statistical distribution will lead to the development of a series of statistical phantoms. Using the Monte Carlo code, the 
organ doses in the phantoms are computed. Then, the organ dose distribution is analyzed and statistical parameters such as the mean value and uncertainty are determined (Xu and Liu, 2011).

The largest sources of uncertainties in internal dose calculations are typically related to the biokinetic model for a radionuclide or radiopharmaceutical and the physical size and shape of subjects and their organs. However, for all ages, one of the major contributors to uncertainty in dose coefficients is the mass. Biokinetic parameters are smaller contributors, suggesting that careful consideration of the knowledge of the mass, as one of the most prominent contributors, should be used to reduce uncertainties (Brill et al., 2006; Stabin, 2008).

Uncertainty in the size and shape of lungs in external radiotherapy was examined by Ebrahimi-Khankook et al. (2014, 2015). In this study, as the first investigation into this subject, statistical phantoms were used for internal dosimetry and the uncertainty related to the lung mass is examined.

\section{Materials and methods}

In this study, the lung as an internal source and its mass as the statistical parameter were selected and a valid method for developing a series of phantoms with statistical lung masses for use in internal dosimetry is proposed. Lung cancer is one of the most prevalent cancers: more than one-quarter of all cancer deaths are due to it. Therefore, it is necessary to treat it. On the other hand, since the vulnerability of the lungs is high, the risk of secondary fatal cancer is high.

It was assumed that the distribution of the lung mass follows the Gaussian normal distribution (Na et al., 2009, 2010) with the average values and standard deviations reported by the International Commission on Radiological Protection (ICRP, 2002). Using a FORTRAN program, 98 random numbers with a Gaussian distribution were created for the lung masses. Volume distributions were obtained by dividing the mass distributions by the lung density $\left(0.352 \mathrm{~g} . \mathrm{cm}^{-3}\right)$ (Han, 2005, p. 57).

In this project, the adult ORNL phantom developed by Cristy and Eckerman was used (Miri-Hakimabad and RafatMotavalli, 2008). This phantom is a hermaphrodite model, which includes both male and female organs. The lung mass (blood and lung tissue) of this phantom has an average value and a standard deviation of $1246 \mathrm{~g}$ and $322 \mathrm{~g}$, respectively (ICRP, 2002).

Finally, 98 phantoms with different lung masses were created and used for the organ dose calculations. The Monte Carlo transport code MCNPX 2.4.0 (Briesmeister, 2000) was used and the dose deposited per source particle (in pGy) was determined with tally F6: $\mathrm{p}$ for a monoenergetic photon internal source with energies of 10, 15, 20, 30, 50, 100, 200, 500, 1000, 1500, 2000 and $4000 \mathrm{keV}$, considering kerma approximation. Using this approximation, it is assumed that the secondary particles are absorbed in the interaction region and just the photon transport is considered. A total of $2 \times 10^{9}$ and almost $1 \times 10^{8}$ particles were tracked for energies lower and higher than $50 \mathrm{keV}$, respectively, to obtain reasonable relative errors due to the Monte Carlo calculation below 3\% for all organs and tissues.
In addition, lung-to-lung S-values (absorbed dose per decay) for the total energy of the emitted electrons, photons, and their sum per decay of ${ }^{131} \mathrm{I}$ were calculated. ${ }^{131} \mathrm{I}$ is a $\beta$-emitting radionuclide, whose emitted electrons can induce tumor necrosis and the long-range gamma ray can be imaged on SPECT for observing the biodistribution of ${ }^{131} \mathrm{I}$ (Zheng et al., 2013). ${ }^{131} \mathrm{I}$-labeled tumor necrosis therapy chimeric antibody $\left({ }^{131} \mathrm{I}-\right.$ chTNT) is used for the treatment of advanced lung cancer in China (Chen et al., 2005; Yu et al., 2006).

It took almost 4 months for the programs to be run and in most cases the relative error was less than $0.1 \%$. However, the values that were calculated with an error of more than $5 \%$ are not mentioned in the tables (the empty spaces in the tables).

For high-energy photons, great differences were observed between the lung dose and the kerma. The discrepancies between the doses of lungs calculated with kerma approximation and with electron tracking at the energies of 500 and $1500 \mathrm{keV}$ were about $36 \%$ and $83 \%$, separately. Therefore, for the photons with energies higher than $500 \mathrm{keV}$, electrons were also tracked and the lung dose was determined using tally F6: e (in P-E mode). However, for the other organs, the absorbed doses were calculated using kerma approximation.

By investigating the statistical changes in the dose due to variations in the lung mass as a source of radiation, the average value and the uncertainty of organ absorbed doses were determined. The uncertainties of organ absorbed doses were determined by standard deviations (SD) of the absorbed doses from the average values. The SD is calculated by the following equation:

$$
S D=\sqrt{\frac{\sum\left(X_{i}-X_{c}\right)^{2}}{N}}
$$

where $X_{i}$ is each value of a statistical dataset, $X_{c}$ is the average value of the statistical dataset and $N$ is the total number of the population.

To compare the deviation of organ absorbed doses from their average values, the coefficient of variation $(\mathrm{CV})$ is calculated, as below:

$$
C V=\frac{S D}{X_{c}}
$$

\section{Results and discussion}

According to the high diffusion of photons, in addition to the source, other organs have an uptake of the dose. In this regard, careful consideration of the effects of changes in the lung mass on the results of the internal dosimetry of the lung and other organs should be made.

\subsection{Monoenergetic photon sources}

The mean absorbed doses normalized to the source energy, specific absorbed fractions (SAFs), for the lung and some organs, together with their CVs, are depicted in Tables 1 and 2, where the "Statistical Data" are SAF mean values and the "Reference Data" are SAFs of the reference phantoms. The discrepancies between our results and those of Cristy and Eckerman are negligible for high energies (less than 1\%) and 
Table 1. Comparison between the SAF mean values and their CVs and those of the reference.

\begin{tabular}{|c|c|c|c|c|c|c|c|c|c|}
\hline \multirow[t]{2}{*}{$\mathbf{E}(\mathbf{k e V})$} & \multicolumn{3}{|c|}{ Lungs } & \multicolumn{3}{|c|}{ Heart Wall } & \multicolumn{3}{|c|}{ Thymus } \\
\hline & $\begin{array}{c}\text { Statistical } \\
\text { Data } \\
\left(\times 10^{-3} \mathrm{~kg}^{-1}\right)\end{array}$ & $\begin{array}{l}\text { CV } \\
(\%)\end{array}$ & $\begin{array}{c}\text { Reference } \\
\text { Data } \\
\left(\times \mathbf{1 0}^{-3} \mathbf{k g}^{-1}\right)\end{array}$ & $\begin{array}{c}\text { Statistical } \\
\text { Data } \\
\left(\times 10^{-3} \mathrm{~kg}^{-1}\right)\end{array}$ & $\begin{array}{l}\text { CV } \\
(\%)\end{array}$ & $\begin{array}{c}\text { Reference } \\
\text { Data } \\
\left(\times 10^{-3} \mathrm{~kg}^{-1}\right)\end{array}$ & $\begin{array}{c}\text { Statistical } \\
\text { Data } \\
\left(\times 10^{-3} \mathrm{~kg}^{-1}\right)\end{array}$ & $\begin{array}{l}\text { CV } \\
(\%)\end{array}$ & $\begin{array}{c}\text { Reference } \\
\text { Data } \\
\left(\times 10^{-3} \mathbf{k g}^{-1}\right)\end{array}$ \\
\hline 10 & 975 & 19 & 781.25 & 0.109 & 38 & 0.0515 & $\ldots \ldots$ & $-\ldots$ & $\ldots$ \\
\hline 15 & 775 & 19 & 641.6 & 3.93 & 19 & 3.28 & 0.014 & 41 & 0.103 \\
\hline 20 & 525 & 19 & 465.63 & 16.9 & 5 & 15.1 & 1.60 & 20 & 1.23 \\
\hline 30 & 243.8 & 14 & 233.3 & 35.8 & 7 & 34.6 & 14.6 & 4 & 12.5 \\
\hline 50 & 90 & 13 & 92.5 & 33.1 & 3 & 33.5 & 20.0 & 0.2 & 19.1 \\
\hline 100 & 51.88 & 12 & 53.13 & 22.6 & 3 & 23.3 & 15.6 & 1 & 15.1 \\
\hline 200 & 51.25 & 13 & 51.88 & 19.9 & 3 & 20.3 & 14.1 & 1 & 13.5 \\
\hline 500 & 51.75 & 12 & 51.38 & 18.5 & 2 & 18.9 & 13.3 & 1 & 12.8 \\
\hline 1000 & 45.44 & 13 & 45.44 & 16.9 & 2 & 17.1 & 12.3 & 1 & 11.8 \\
\hline 1500 & 38.96 & 12 & 39.67 & 15.5 & 2 & 15.7 & 11.3 & 1 & 10.9 \\
\hline 2000 & 33.66 & 12 & 34.78 & 14.5 & 2 & 14.6 & 10.6 & 1 & 10.2 \\
\hline 4000 & 20.83 & 9 & 22.83 & 11.8 & 2 & 12.0 & 8.72 & 1 & 8.41 \\
\hline
\end{tabular}

Table 2. Comparison between the SAF mean values and their CVs and those of the reference.

\begin{tabular}{|c|c|c|c|c|c|c|c|c|c|}
\hline $\mathrm{E}(\mathrm{keV})$ & & ophag & & & hyroi & & & drena & \\
\hline & $\begin{array}{c}\text { Statistical } \\
\text { Data } \\
\left(\times 10^{-3} \mathrm{~kg}^{-1}\right)\end{array}$ & $\begin{array}{l}\text { CV } \\
(\%)\end{array}$ & $\begin{array}{c}\text { Reference } \\
\text { Data } \\
\left(\times \mathbf{1 0}^{-3} \mathbf{k g}^{-1}\right)\end{array}$ & $\begin{array}{c}\text { Statistical } \\
\text { Data } \\
\left(\times 10^{-3} \mathrm{~kg}^{-1}\right)\end{array}$ & $\begin{array}{l}\text { CV } \\
(\%)\end{array}$ & $\begin{array}{c}\text { Reference } \\
\text { Data } \\
\left(\times 10^{-3} \mathbf{k g}^{-1}\right)\end{array}$ & $\begin{array}{c}\text { Statistical } \\
\text { Data } \\
\left(\times 10^{-3} \mathbf{k g}^{-1}\right)\end{array}$ & $\begin{array}{l}\text { CV } \\
(\%)\end{array}$ & $\begin{array}{c}\text { Reference } \\
\text { Data } \\
\left(\times 10^{-3} \mathbf{k g}^{-1}\right)\end{array}$ \\
\hline 10 & $-\ldots$ & -- & $-\ldots$ & $-\ldots$ & -- & $-\ldots$ & $-\cdots$ & --- & $-\ldots$ \\
\hline 15 & 0.32 & 44 & 0.223 & 0.003 & 86 & - - - - - & 0.7 & 39 & 0.360 \\
\hline 20 & 6.28 & 23 & 4.72 & 0.126 & 44 & 0.083 & 3.34 & 59 & 3.50 \\
\hline 30 & 23.3 & 8 & 20.1 & 3.13 & 20 & 2.25 & 11.6 & 22 & 11.6 \\
\hline 50 & 24.9 & 2 & 23.5 & 7.43 & 10 & 5.99 & 13.0 & 10 & 13.5 \\
\hline 100 & 19.2 & 0.5 & 18.6 & 6.25 & 5 & 5.48 & 10.8 & 6 & 11.3 \\
\hline 200 & 17.6 & 0.4 & 17.0 & 5.63 & 6 & 4.81 & 10.1 & 6 & 10.6 \\
\hline 500 & 16.4 & 1 & 15.8 & 5.38 & 6 & 4.64 & 9.59 & 6 & 10.1 \\
\hline 1000 & 14.8 & 1 & 14.3 & 5.13 & 5 & 4.43 & 8.81 & 6 & 9.31 \\
\hline 1500 & 13.5 & 1 & 13.1 & 4.79 & 5 & 4.17 & 8.08 & 6 & 8.58 \\
\hline 2000 & 12.6 & 1 & 12.2 & 4.53 & 5 & 3.97 & 7.50 & 6 & 8.00 \\
\hline 4000 & 10.2 & 1 & 9.94 & 3.84 & 5 & 3.38 & 6.14 & 5 & 6.59 \\
\hline
\end{tabular}

up to $7 \%$ for low energies (Cristy and Eckerman, 1987a). The difference for low energies is due to the deviations in the lung mass of the incorporated phantoms.

\subsubsection{Statistical changes in the lung SAF}

According to the results obtained, the lung, which is the gamma source, received the maximum dose. For energies higher than $500 \mathrm{keV}$, increasing the energy increases the lung SAFs, but there is no specific trend for lower energies. For instance, for gamma energy of $50 \mathrm{keV}$, the SAF $\left(90 \times 10^{-3} \mathrm{~kg}^{-1}\right)$ is less than that at energy of $10 \mathrm{keV}\left(975 \times 10^{-3} \mathrm{~kg}^{-1}\right)$. This could be explained by considering the direct relation between the dose $(D)$, mass attenuation coefficient $(\mu)$, organ-source distance $(x)$ and mean energy of the source $(\bar{E})$, as below:

$$
D \infty \mu x . \bar{E} \text {. }
$$

According to the dose and the mass relationship, as the lung mass increases, the lung absorbed dose decreases. To evaluate the rate of this reduction for each energy level, the SAF as a function of the inverse of the mass (mass ${ }^{-1}$ ) and SAF as a function of the inverse of the surface (mass ${ }^{-\frac{2}{3}}$ ) in terms of the lung mass were plotted in Figure 1. To have a precise comparison, mass $^{-1}$ and mass ${ }^{-\frac{2}{3}}$ figures were multiplied at a constant value to fit their start point with the SAF. The changes in the lung surface are almost equal to mass ${ }^{\frac{2}{3}}$ (lungs can be considered as two half-ellipsoids). In the mass distribution of the lung, the changes in the inverse of the mass and the surface of the largest lung to the smallest lung are almost $70 \%$ and $56 \%$, respectively. As expected, increasing the lung size decreases the ratio of the lung surface to the lung mass.

The SAF reduction of the largest lung relative to the smallest lung at energy of $10 \mathrm{keV}$ is $69 \%$, which is almost proportional to the variations in the inverse of the lung mass, 


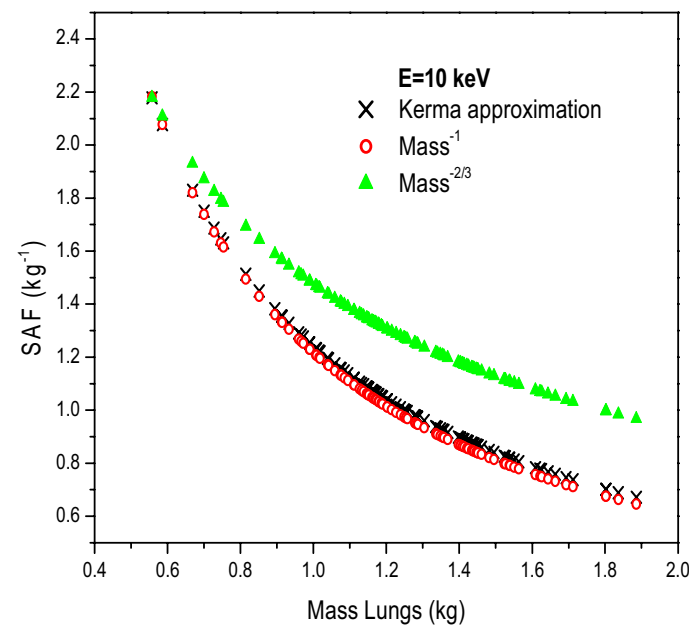

(a)

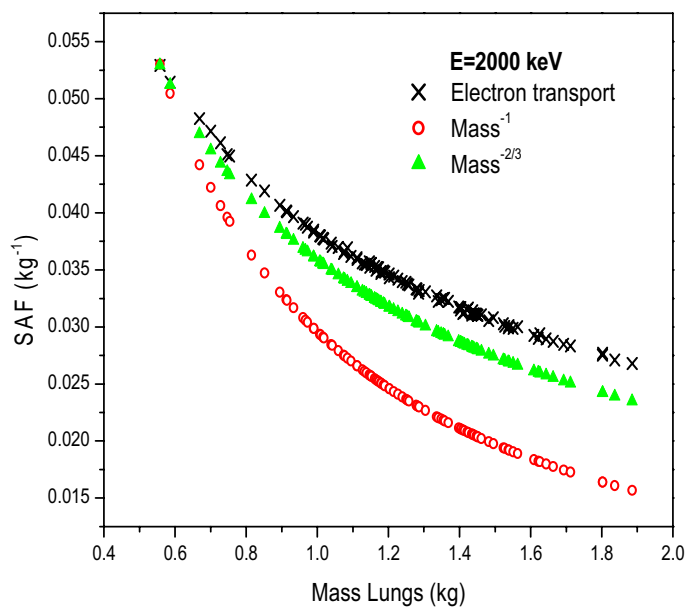

(c)

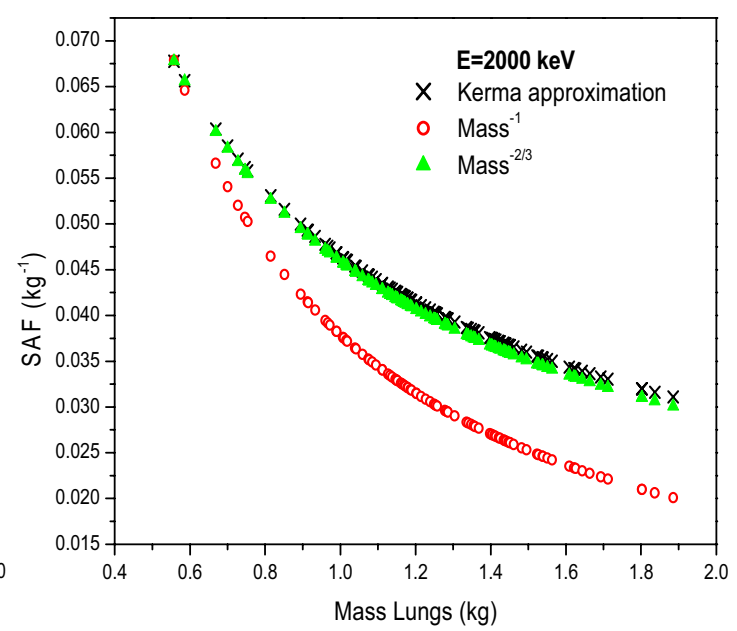

(b)

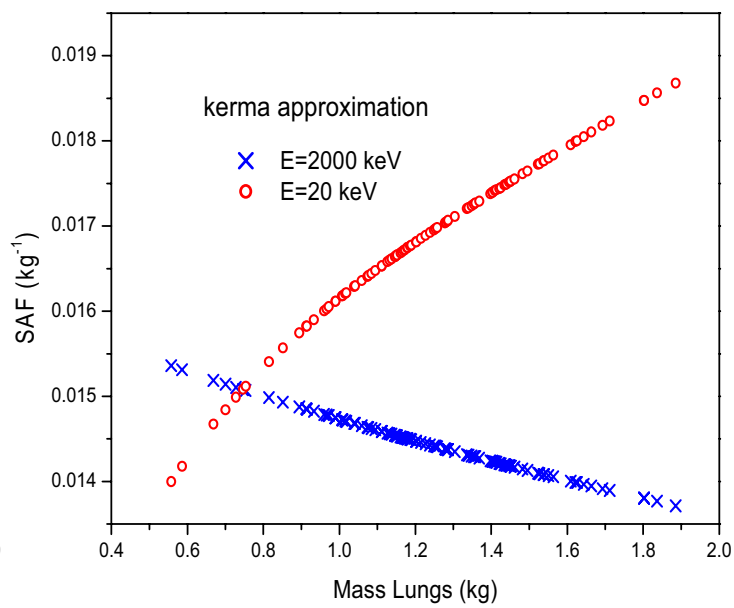

(d)

Figure 1. (a) Lung SAFs with kerma approximation as a function of mass for energies of $10 \mathrm{keV}$, (b) $2000 \mathrm{keV}$ and (c) $2000 \mathrm{keV}$ including electron transport; (d) heart wall SAFs for energies of 20 and $2000 \mathrm{keV}$ by kerma approximation.

consistent with the agreement between the SAF and the mass ${ }^{-1}$ curves in Figure 1a. The rate of this reduction decreases with increasing energy. If the lung size increases, the probability of trapping and, as a result, the interaction of high-energy and high-range photons in the lung increases. For instance, at energy of $2000 \mathrm{keV}$, using kerma approximation, the rate of SAF reduction of the largest lung relative to the smallest lung is almost $54 \%$, consistent with the SAF and mass ${ }^{-2 / 3}$ curves (Figure $1 \mathrm{~b}$ ); it is almost proportional to the inverse of the lung surface. In addition, at high energies, the dominant interaction of photons with soft tissue is Compton scattering, which produces secondary electrons. On the other hand, increasing the lung size increases the chance for secondary particles to return to the lung; this leads to a lower reduction rate for the SAF compared with kerma. Therefore, if an electron is tracked, the SAF of the largest lung relative to the smallest lung decreases by almost $49 \%$ for gamma energy of $2000 \mathrm{keV}$ (see Figure 1c). According to Figures $1 \mathrm{~b}$ and $1 \mathrm{c}$, changes in the lung SAF are not proportional to changes in the lung mass.
For photons, Snyder of Oak Ridge National Laboratory showed that the photon specific absorbed fractions vary directly with the two-thirds power of the mass for self-irradiation (i.e., source $=$ target) if the photon mean path length is long, compared with the organ diameter (Snyder, 1970; Stabin et al., 2005).

Our calculations are representative of the fact that for photons, SAFs do not always change directly with the two-thirds power of the mass for the lung as the source, but the power ranges from $\frac{1}{2}$ to 1 at energies between 10 and $4000 \mathrm{keV}$ (i.e., $\frac{1}{2}$ for $4000 \mathrm{keV}$ and 1 for $10 \mathrm{keV}$ ).

\subsubsection{Statistical changes in the other organ SAFs}

Besides the lung, the organs whose centroids are located closer to the source (Cristy and Eckerman, 1987b) (in order from closest to farthest: the heart wall, esophagus, thymus, breast, adrenal, spleen and thyroid) absorb a significant dose 


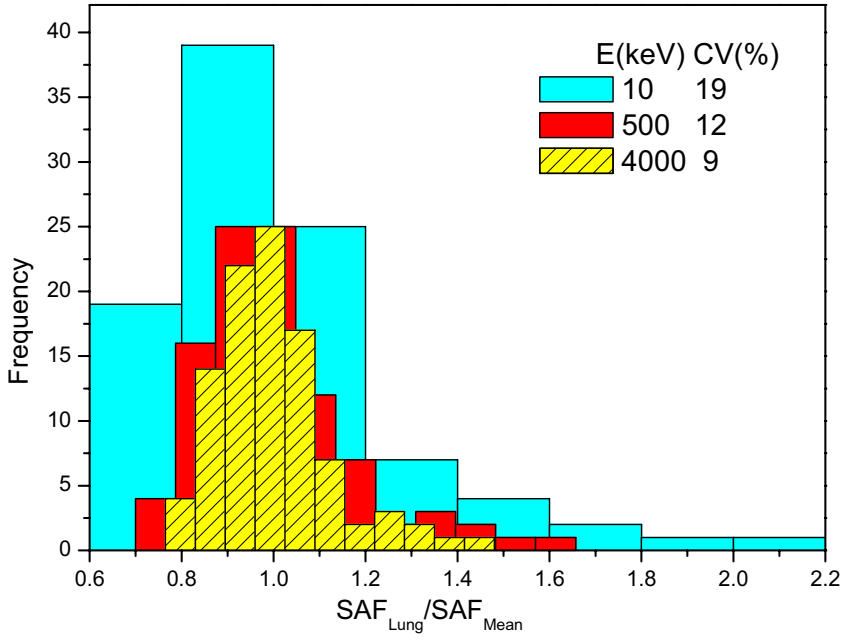

Figure 2. Statistical distribution of lung SAFs at three different photon energies.

among other target tissues. Enhancing the lung size decreases its distance from the other organs, and thus causes them to receive higher doses. At low energies, the SAFs of the organs located very close to the lung, such as the heart wall and thymus, increase with the lung mass. This is due to the absorption of low-energy and short-range photons. In this situation, a reduction in the distances between the source and target organs enhances the possibility of gamma-ray interactions in these organs. In addition, at low energies the occurrence of photoelectric effects is dominant, which causes an increase in the organ SAF.

By increasing the photon energy, the enhancement of the SAF rate declines. As expected, high diffusion of high-energy photons leads to a reduction of the photon interactions in these organs. For example, the heart wall SAF at energy of $20 \mathrm{keV}$ in the largest lung to the smallest lung increases by $33 \%$. This rate reduces with increasing energy and for energies higher than $30 \mathrm{keV}$, this trend is reversed, so that the SAF decreases. For instance, the heart wall SAF in the largest lung to the smallest lung decreases by $11 \%$ at the energy of $2000 \mathrm{keV}$ (see Figure 1d).

The SAFs of the other organs such as the thyroid, intestine, gall bladder, etc. increase as the organ-source distance decreases (for each energy).

\subsubsection{Estimating the statistical parameters of the lung SAF}

Figure 2 displays the Gaussian distribution of statistical changes in the lung SAFs at three different photon energies ( $E$ is photon energy). In this histogram, the lung specific absorbed fractions $\left(\mathrm{SAF}_{\text {lung }}\right)$ are in units of $\mathrm{kg}^{-1}$ per particle of the source, which were normalized to 1 by dividing these values by the average value of the lung $\mathrm{SAF}\left(\mathrm{SAF}_{\mathrm{Mean}}\right)$. The vertical axis is representative of the number of subjects (frequency) and the $\mathrm{CV}$ shows the percent of deviation of the SAF from the average value. It is evident from the data that increasing the photon energy decreases the width of the Gaussian distribution, so that at higher energies the deviation is smaller.

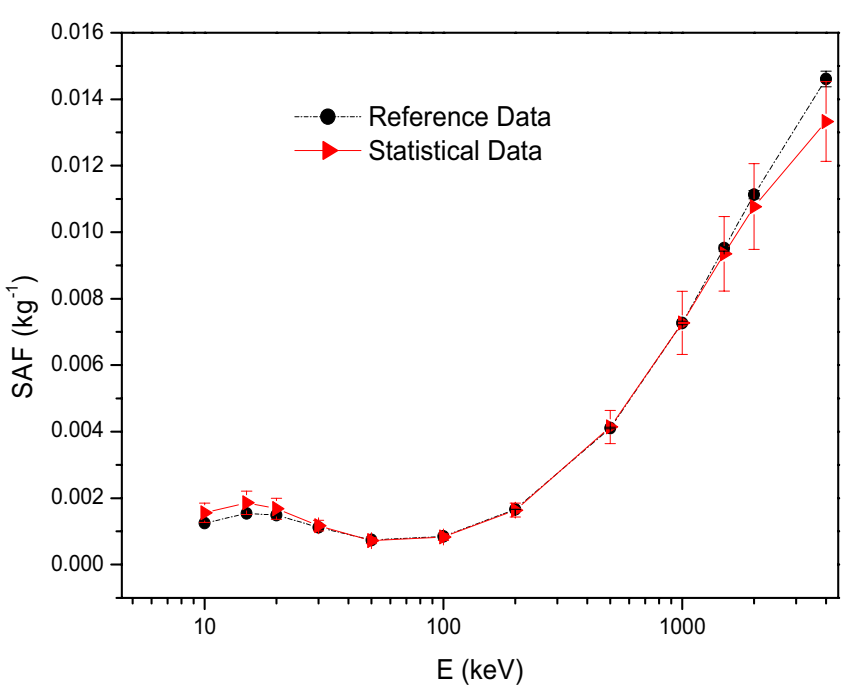

Figure 3. Comparison between the lung reference and statistical SAFs in terms of photon energy.

The CV of the lung at energy of $10 \mathrm{keV}$ is $19 \%$, while at energy of $4000 \mathrm{keV}$, it decreases to $9 \%$ (see Figure 2 and the CVs for the other energies in Table 1). It should be noted that CV values higher than $5 \%$ are considerable. Therefore, changing the mass has significant effects on the SAFs of the lung, especially for low-energy photons.

By comparing the results of the statistical SAF with those of the reference for the lung (see Table 1), it is observed that the reference data is almost in the range of statistical data (i.e. $\left.X_{c} \pm S D\right)$.

For further investigations into reference and statistical values of the lung SAF, their related curves in terms of source energy are plotted in Figure 3. In this figure SDs related to the statistical errors due to the lung mass scattering are also observed. In this research, the uncertainty consists of the relative statistical uncertainty due to the Monte Carlo calculations and the standard deviation (SD) arising from the lung mass variation. Since the statistical error due to the Monte Carlo calculations is much less than that of the lung mass scattering, the total standard deviation would be almost equal to the standard deviation due to the lung mass scattering. It can also be observed that the statistical data are higher than the reference data in the low-energy region and lower in the high-energy region. The reason for this behavior is that the discrepancy between the average value of the SAF for larger and smaller lungs, than the reference lung, with the reference data is different. They are almost equal to the reference data for the energy of $20-2000 \mathrm{keV}$.

\subsubsection{Estimating the statistical parameters of the other organ SAFs}

Figure 4 displays the Gaussian distribution of the the statistical changes in thyroid SAFs for three different photon energies. As observed, increasing the photon energy decreases the width of the Gaussian distribution, so that at higher energies, the deviation is smaller. The $\mathrm{CV}$ for the thyroid at energy of $20 \mathrm{keV}$ is $44 \%$, and it decreases to $5 \%$ at energy of $4000 \mathrm{keV}$. 
Table 3. Comparison of the organ SAFs relative to the lung SAF (in percent).

\begin{tabular}{lccccccccccccc}
\hline E $(\mathbf{k e V})$ & $\mathbf{1 0}$ & $\mathbf{1 5}$ & $\mathbf{2 0}$ & $\mathbf{3 0}$ & $\mathbf{5 0}$ & $\mathbf{1 0 0}$ & $\mathbf{2 0 0}$ & $\mathbf{5 0 0}$ & $\mathbf{1 0 0 0}$ & $\mathbf{1 5 0 0}$ & $\mathbf{2 0 0 0}$ & $\mathbf{4 0 0 0}$ \\
\hline Heart Wall & 0 & 1 & 3 & 15 & 37 & 44 & 39 & 36 & 37 & 40 & 43 & 57 \\
Esophagus & 0 & 0 & 1 & 10 & 28 & 37 & 34 & 32 & 33 & 35 & 37 & 50 \\
Thymus & 0 & 0 & 0 & 6 & 22 & 30 & 27 & 26 & 27 & 29 & 31 & 42 \\
Adrenals & 0 & 0 & 1 & 5 & 14 & 21 & 20 & 21 & 19 & 21 & 22 & 30 \\
Spleen & 0 & 0 & 1 & 4 & 13 & 16 & 15 & 14 & 14 & 16 & 17 & 23 \\
Pancreas & 0 & 0 & 0 & 2 & 9 & 14 & 13 & 12 & 12 & 13 & 15 & 20 \\
Thyroid & 0 & 0 & 0 & 1 & 8 & 12 & 11 & 11 & 11 & 12 & 13 & 19 \\
ET Region & 0 & 0 & 0 & 1 & 6 & 8 & 8 & 8 & 9 & 10 & 11 & 15 \\
Gall bladder & 0 & 0 & 0 & 0 & 4 & 7 & 7 & 7 & 7 & 8 & 9 & 12 \\
Kidney & 0 & 0 & 0 & 0 & 3 & 6 & 6 & 6 & 7 & 8 & 9 & 12 \\
Salivary Gland & 0 & 0 & 0 & 0 & 3 & 5 & 5 & 5 & 6 & 6 & 7 & 10 \\
Skin & 0 & 0 & 0 & 1 & 3 & 4 & 4 & 4 & 5 & 6 & 6 & 9 \\
Brain & 0 & 0 & 0 & 0 & 0 & 1 & 1 & 1 & 2 & 2 & 3 & 4 \\
\hline
\end{tabular}

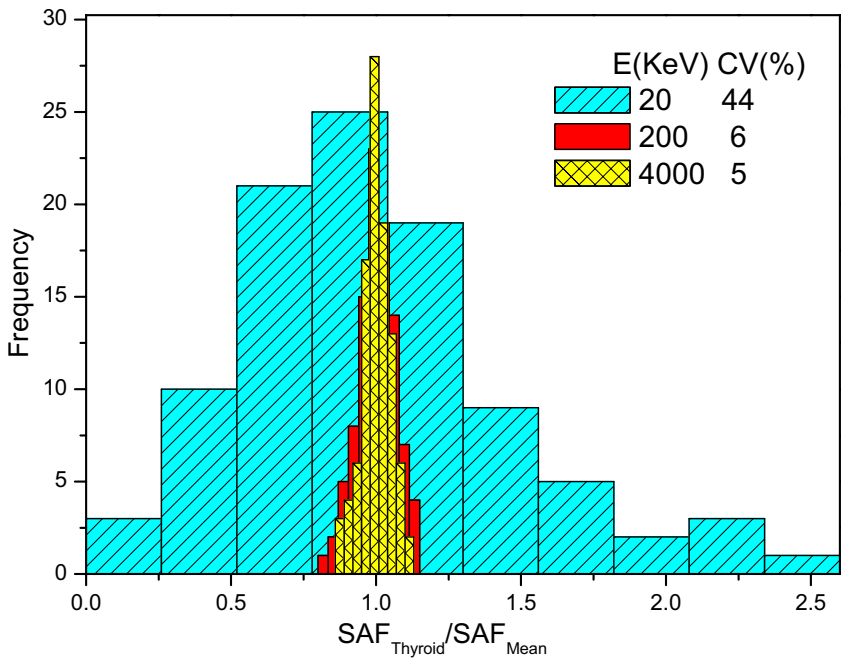

Figure 4. Statistical distribution of thyroid SAFs at three different photon energies.

The SAFs and CVs for some organs are listed in Tables 1 and 2 and compared with those of the reference.

Considering the $\mathrm{CV}$ values reported in the tables, for organs located close to the source such as the heart wall, regarding their low SAF at low energies and their low $\mathrm{CV}$ at high energies, the effect of variations in lung masses on the SAF is negligible. On the other hand, for organs located further from the source such as the colon, intestine and gall bladder, with respect to their low SAF at all energies, the effect of changes in the lung mass is marginal. For instance, the heart wall SAF at $15 \mathrm{keV}$ is $1 \%$ of the lung SAF, which is a very small amount, and thus its CV, though standing at $19 \%$, is negligible. The SAF for cross-irradiation (i.e., source $\neq$ target) does not change with differences in mass, provided the source and target are sufficiently separated and that the change in mass of one or both does not appreciably change the relative geometry (Stabin et al., 2005).

Since the source organ (lung) absorbs the maximum dose, the other organ SAFs were compared with the lung SAF (see Table 3). Relative values of more than $20 \%$ were considered noticeable and values less than $0.5 \%$ are shown as 0 . Therefore, the importance of performing statistical calculations is not equal for all organs and the amount of the dose received by each organ determines this issue.

\subsection{Decay of ${ }^{131}$ I}

\subsubsection{Estimating the statistical parameters of the lung SAF}

In internal dosimetry, photons are only incorporated for diagnostic purposes. Since the injected doses for diagnosis are low enough, the changes and therefore the uncertainty are marginal. With respect to implementing high doses for therapeutic purposes, determining uncertainty regarding the lung mass changes is of vital importance. Since an overwhelming majority of the electron energy will be absorbed within the source tissue, it is sufficient to consider the effects of the lung mass changes on the SAF of the lung and no other organs. Therefore, in this research, the uncertainty for the lung per decay of ${ }^{131}$ I was calculated. Our results, consistent with previous studies (Williams et al., 2002; Stabin et al., 2005), are demonstrative of the fact that for $\beta$-emissions, the dose (and $\mathrm{S}$-value) is inversely proportional to the mass.

The figure for lung-to-lung $\mathrm{S}$-values and $\mathrm{CV}$ s for total energy emitted as electrons, photons and their sum per decay of ${ }^{131} \mathrm{I}$ were calculated, $0.0320 \mathrm{pGy} \cdot \mathrm{Bq}^{-1} \cdot \mathrm{s}^{-1} ; 21 \%$, $0.0035 \mathrm{pGy} \cdot \mathrm{Bq}^{-1} \cdot \mathrm{s}^{-1} ; 11 \%, 0.0358 \mathrm{pGy} \cdot \mathrm{Bq}^{-1} \cdot \mathrm{s}^{-1} ; 18 \%$ for electrons, photons and their sum, respectively. S-value histograms are depicted in Figure 5. Since the contribution of the photons to the $\mathrm{S}$-value of ${ }^{131} \mathrm{I}$ is negligible, the $\mathrm{S}$-value of ${ }^{131} \mathrm{I}$, like $\beta$-emissions, is inversely proportional to the mass. To correct the $S$-values, the figures were multiplied by the lung mass, and the results are shown in Figure 6. It can be seen from the figure that regarding the mass correction for S-values, the histogram has been sharpened and the $\mathrm{CV}$ declined significantly from $18 \%$ to $1 \%$.

There was a $6 \%$ difference between the S-value per decay of ${ }^{131}$ I calculated in this study and the figure calculated by Snyder et al. (1975). This could be due to the discrepancy in the lung mass of the phantom used in this study and that of the Snyder study. 


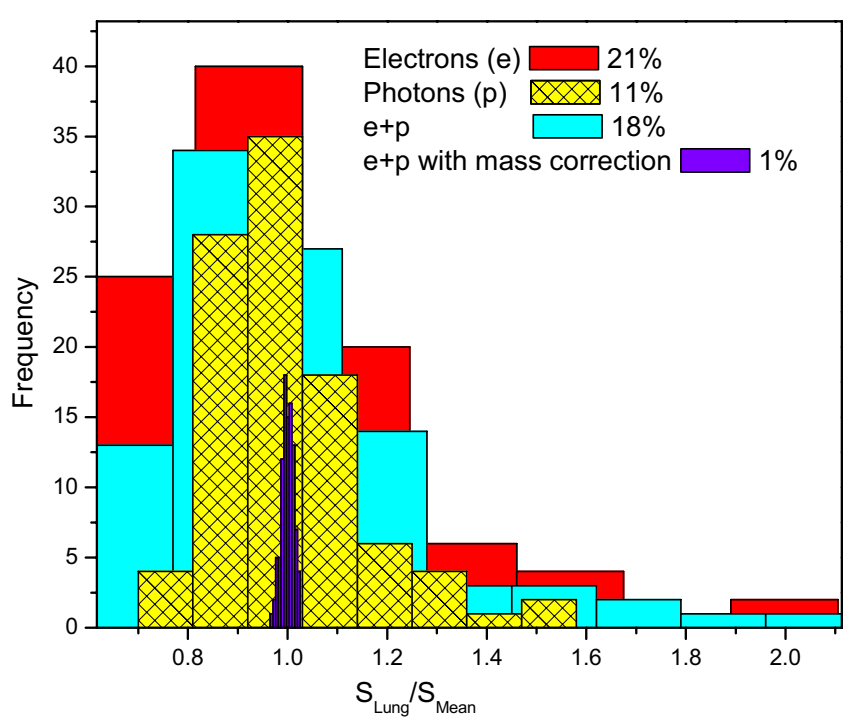

Figure 5. Statistical distribution of the S-value per decay of ${ }^{131} \mathrm{I}$ for photons (p), electrons (e), e+p and $\mathrm{e}+\mathrm{p}$ with mass correction with their CVs.

\subsubsection{Estimating the risk of a secondary radiation-induced cancer}

${ }^{131} \mathrm{I}$-chTNT is a radiopharmaceutical used for the treatment of advanced lung cancer. In previous studies, some patients received 2 doses of $0.8 \mathrm{mCi} \cdot \mathrm{kg}^{-1}$ of body weight administered 2 weeks apart (Chen et al., 2005; Yu, et al., 2006). The average absorbed dose for normal lung tissue was $2.35 \mathrm{~Gy}$ (Chen et al., 2005). According to the coefficient of variation of $18 \%$ of the absorbed dose, the SD for the average absorbed dose of $2.35 \mathrm{~Gy}$ will be equal to $0.42 \mathrm{~Gy}$, which is noticeable. The probability of fatal secondary malignancies associated with radiation therapy of the lung stands at $0.85 \%$ per Sv (Kry et al., 2005). For a SD of 0.42 Gy (considering the tissue weight coefficient equals $0.0504 \mathrm{~Sv}$ for the lung), the probability of fatal cancer was found to be as high as $0.043 \%$ for each injected dose. To diminish this risk, the administered activity should be multiplied by the patient's lung mass. The determination of lung mass is easily made using morphological imaging (for example, computed tomography, magnetic resonance tomography, etc.) and Siemens' Volume Evaluation (VE) version B10/2004A software (Sylvan, 2005) or other relevant software. Thus, anatomic imaging is required. This is considered suitable and cost-effective since we are dealing with cancer patients and these data are usually available.

\section{Conclusion}

In this study, as far as we know the first investigation into internal dosimetry, a library of dose values and uncertainties related to the dispersion of lung masses was obtained. These data help physicians to ensure the accuracy of data used for the dose estimation of different individuals. It was observed that the source organ mass, photon energy and the distance between the source and the target organs affect the value of the
SAF. According to the results, the SAF of the lung depends on its mass, while the amount of the SAF of other organs depends on their distances from the source. For photons, the lung CV values changed between $9 \%$ (at $4000 \mathrm{keV}$ ) and $19 \%$ (at $10 \mathrm{keV}$ ).

With regard to incorporation of high doses for therapeutic purposes, determining uncertainty regarding the lung mass changes is of great importance. Hence, the CV per decay of ${ }^{131}$ I was calculated, standing at $18 \%$, which decreased to $1 \%$ after the correction of the mass. The major contributors to this $\mathrm{CV}$ were the electrons.

It is noteworthy that for electrons resulting from the decay of ${ }^{131} \mathrm{I}$, the dose (and $\mathrm{S}$ ) is inversely proportional to the mass, while the dose (and SAF) for photons changes with the power, ranging from $\frac{1}{2}$ to 1 at energies between 10 and $4000 \mathrm{keV}$ (i.e., $\frac{1}{2}$ for $4000 \mathrm{keV}$ and 1 for $10 \mathrm{keV}$ ).

As a result, changes in the mass of the lung are significant for calculating absorbed doses of the lung (as the source), while for other organs, they are negligible. In future studies, the effects of statistical changes in the lung and other source organs on the dose will be investigated for other reference phantoms (which are more similar to the human body) and the amount of uncertainty in using reference phantoms for dose estimation will be further discussed.

\section{References}

Briesmeister J.F. (2000) A general Monte Carlo N-particle transports code: version4c. Report LA-13709-M, 1-427, Los Alamos National Laboratory, Los Alamos.

Brill A.B., Stabin M., Bouville A., Ron E. (2006) Normal organ radiation dosimetry and associated uncertainties in nuclear medicine, with emphasis on iodine-131, Radiat. Res. 166 (1), 128-140.

Chen S., Yu L., Jiang C., Zhao Y., Sun D., Li S., Ju D.W. (2005) Pivotal Study of Iodine-131 - Labeled Chimeric Tumor Necrosis Treatment Radioimmunotherapy in Patients With Advanced Lung Cancer, J. Clin. Oncol. 23 (7), 1538-1547.

Cristy M., Eckerman K.F. (1987a) Specific absorbed fractions of energy at various ages from internal photon sources. VII. Adult Male. ORNL/TM-8381/V7. Oak Ridge National Laboratory, Tennessee.

Cristy M., Eckerman K.F. (1987b) Appendices B, C, D. In: Specific absorbed fractions of energy at various ages from internal photon sources. I. Methods. ORNL/TM-8381/V1. Oak Ridge National Laboratory, Tennessee.

Ebrahimi-Khankook A., Miri-Hakimabad H., Rafat-Motavalli L. (2014) Studying the effect of the lung size variation on dosimetry and systematic parameters of FUM-IVNAA facility, $J$. Radioanal. Nucl. Chem. 303, 2263-2270.

Ebrahimi-Khankook A., Miri-Hakimabad H., Rafat-Motavalli L. (2015) A study of the effect of the lung shape on the lung absorbed dose in six standard photon and neutron exposure geometries, Radioprotection 50 (1), 65-72.

Han E.Y. (2005) Revised Series of Stylized Anthropometric Phantoms for Internal and External Radiation Dose Assessment, Ph.D Thesis, The University of Florida, pp. 16-36, 239-240.

ICRP Publication 23 (1975) International Commission on Radiological Protection. Report of the Task Group on Reference Man. 
ICRP Publication 89 (2002) International Commission on Radiological Protection. Basic Anatomical and Physiological Data for Use in Radiological Protection: Reference Values. Pergamon, Oxford.

Kry S.F., Salehpour M., Followill D.S., Stovall M., Kuban D.A., White R.A., Rosen I.I. (2005) The calculated risk of fatal secondary malignancies from intensity-modulated radiation therapy, Int. J. Radiat. Oncol. Biol. Phys. 62 (4), 1195-1203.

Miri-Hakimabad H., Rafat-Motavalli, L. (2008) Evaluation of specific absorbed fractions from internal photon sources in ORNL analytical adult Phantom, Radiat. Prot. Dosim. 128 (4), 427-431.

Mofrad F.B., Zoroofi R.A., Tehrani-Fard A.A., Akhlaghpoor S., Hori M., Chen Y.W., Sato Y. (2010) Statistical construction of a Japanese male liver phantom for internal radionuclide dosimetry, Radiat. Prot. Dosim. 141 (2), 140-148.

Na Y, Zhang J, Xu G, Hang B., Caracappa PF (2009) Next-generation deformable patient modeling for Monte Carlo assessment of organ doses Med. Phys. 362783

Na Y.H., Zhang B., Zhang J., Caracappa P.F., Xu X.G. (2010) Deformable adult human phantoms for radiation protection dosimetry: anthropometric data representing size distributions of adult worker populations and software algorithms, Phys. Med. Biol. 55 (13), 3789-3812.

Snyder W.S. (1970) Estimates of absorbed fraction of energy from photon sources in body organs (No. CONF-691212). Oak Ridge National Laboratory, Tennessee.

Snyder W.S., Ford M.R., Warner G.G., Watson S.B. (1975) "S" Absorbed dose per unit cumulated activity for selected radionuclides and organs. MIRD pamphlet no. 11. The Society of Nuclear Medicine, New York.
Stabin M.G. (2008) Uncertainties in internal dose calculations for radiopharmaceuticals. J. Nucl. Med. 49 (5), 853-860.

Stabin M.G. (2013) Internal dosimetry in nuclear medicine, Braz. J. Radiat. Sci. 01, 1-15.

Stabin M.G., Sparks R.B., Crowe, E. (2005) OLINDA/EXM: the second-generation personal computer software for internal dose assessment in nuclear medicine, J. Nucl. Med. 46 (6), 1023-1027.

Sylvan E. (2005) CT-based measurement of lung volume and attenuation of deceased, http://urn.kb.se/resolve?urn\$=\$urn:nbn:se:liu: diva-4417.

Williams L.E., Liu A., Yamauchi D.M., Lopatin G., Raubitschek A.A., Wong J.Y. (2002) The two types of correction of absorbed dose estimates for internal emitters. Cancer 94 (S4), 1231-1234.

Xu X.G., Liu T. (2011) Quantifying Uncertainty in Radiation Protection Dosimetry Using Statistical Phantoms. In: The Third International Workshop on Computational Phantoms for Radiation Protection, Imaging and Radiotherapy, August 8-9, Tsinghua University, Beijing.

Xu X.G., Eckerman K.F. (Eds.) (2009) Handbook of anatomical models for radiation dosimetry. CRC Press.

Yu L., Ju D.W., Chen W., Li T., Xu Z., Jiang C., Epstein, A.L. (2006) ${ }^{131}$ I-chTNT radioimmunotherapy of 43 patients with advanced lung cancer, Cancer Biotherapy $\mathcal{E}$ Radiopharmaceuticals 21 (1), $5-14$.

Zheng S.G., Xu H.X., Lu M.D., Yue D.C., Xie X.Y., Liu G.J. (2013) Radiofrequency Ablation Before Intratumoral Injection of ${ }^{131} \mathrm{I}$ chTNT Improves the Tumor-to-Normal Tissue Ratio in Solid VX2 Tumor, Cancer Biotherapy and Radiopharmaceuticals 28 (10), 725-730.

Cite this article as: Z. Sajjadi, H. Miri-Hakimabad, L. Rafat-Motavalli, S. Mohammadi. Evaluating the effects of statistical changes on internal dosimetry. Radioprotection 50(3), 187-194 (2015). 